\title{
Forecasting Parking Lots Availability: Analysis from a Real-World Deployment
}

\author{
Manuele Barraco*, Nicola Bicocchi*‡, Marco Mamei ${ }^{\dagger \ddagger}$, Franco Zambonelli ${ }^{\dagger \ddagger}$ \\ *Dipartimento di Ingegneria "Enzo Ferrari" \\ $\dagger^{\dagger}$ Dipartimento di Scienze e Metodi dell'Ingegneria \\ $\ddagger$ Artificial Intelligence Research and Innovation Center AIRI \\ University of Modena and Reggio Emilia, Italy \\ Email: name.surname@unimore.it
}

\begin{abstract}
Smart parking technologies are rapidly being deployed in cities and public/private places around the world for the sake of enabling users to know in real time the occupancy of parking lots and offer applications and services on top of that information. In this work, we detail a real-world deployment of a full-stack smart parking system based on industrial-grade components. We also propose innovative forecasting models (based on CNN-LSTM) to analyze and predict parking occupancy ahead of time. Experimental results show that our model can predict the number of available parking lots in a $\pm 3 \%$ range with about $80 \%$ accuracy over the next 1-8 hours. Finally, we describe novel applications and services that can be developed given such forecasts and associated analysis.
\end{abstract}

\section{INTRODUCTION}

Every year more than 50 millions new vehicles hit public roads. Over the long term, this increases both the likelihood of disruptions of the traffic flow and consequent pollution but also increases significantly the time spent in search for parking spots. According to [1], the search of free parking spaces is responsible for up to $40 \%$ of the overall traffic within US cities. Similar phenomena can be observed in large cities all over the world. If fact, instead of being informed on the number and location of available spots, drivers rely on on-road perceptions, empirical tries, and past-experience.

Due to these reasons, smart parking technologies have been recently developed. These solutions rely on a complex blend of hardware, software and communication technologies aiming at different trade-offs depending on the desired outcome [2], [3]. These systems, generally, involve sensing the state of parking spots, and distribute the collected data to the final users by leveraging cloud-based architectures for the sake of reducing drivers frustration and improving traffic flows. More recently, learning techniques have been applied to the field in various forms (refer to [4] for a complete review), spanning from improving the sensors precision, developing vision-based sensors [5], [6], [7], crowd-sourcing [8], to predicting the availability of parking lots [9], [10], [11], [12].

Smart parking services based on parking prediction allow drivers to organize their transports before departures or during their trips, and even they could provide the possibility of booking a parking spot in advance. Furthermore, predicting parking lots availability, in addition to enabling drivers to take more informed decisions, can also pave the way to additional services such as pricing lots in a dynamic fashion based on the future states of the parking system and traffic flows. These mechanisms, set aside the likely increment in profitability of parking providers, can also be viewed as social feedback loops improving the ability of large heterogeneous organisms such as smart cities to self-regulate themselves.

In this context, the contribution of this work is twofold. Firstly, we describe a full-stack implementation of a smart parking system installed in an Italian mid-sized city center. Secondly, we apply state-of-the-art deep learning, convolutional neural networks to the time series collected by the sensors to forecast parking availability in real time. Results shows that the proposed approach can successfully predict the availability of parking lots over the next 8 hours.

The remainder of this paper is organized as follows. In Section II we describe the architecture used for data collection and computation. In Section III, we discuss two families of prediction techniques (i.e., statistical and deep neural networks) that have been applied. In Section IV we discuss innovative services that can be enabled by timely and accurate predictions of the available parking spaces. Finally, Section $\mathrm{V}$ discusses related work and Section VI draws concluding remarks.

\section{DEPLOYMENT}

\section{A. IoT Architecture}

The data collection infrastructure has been deployed in the city center of a mid-sized Italian city (Mantova). It is a cloud-based IoT architecture routing the messages from the sensors to several cloud-based application servers processing the data and exposing results via REST interfaces. The overall architetecture is depicted in Figure 1.

Concerning the sensor layer, 70 Bosch Parking Lot sensors have been installed in a straight line in a crowded street of the center. The sensors send MQTT packets over a low energy LoRaWAN channel to a single Cellnext gate covering an approximate radius of $5 \mathrm{~km}$. MQTT packets are then routed to an instance of the PtNetSuite network server. It is a Linuxbased, modular server that can be regarded as the backbone of the whole LoRaWAN network. The network infrastructure, comprising the LoraWAN gate and the access to the network server has been provided by the A2A Group. 

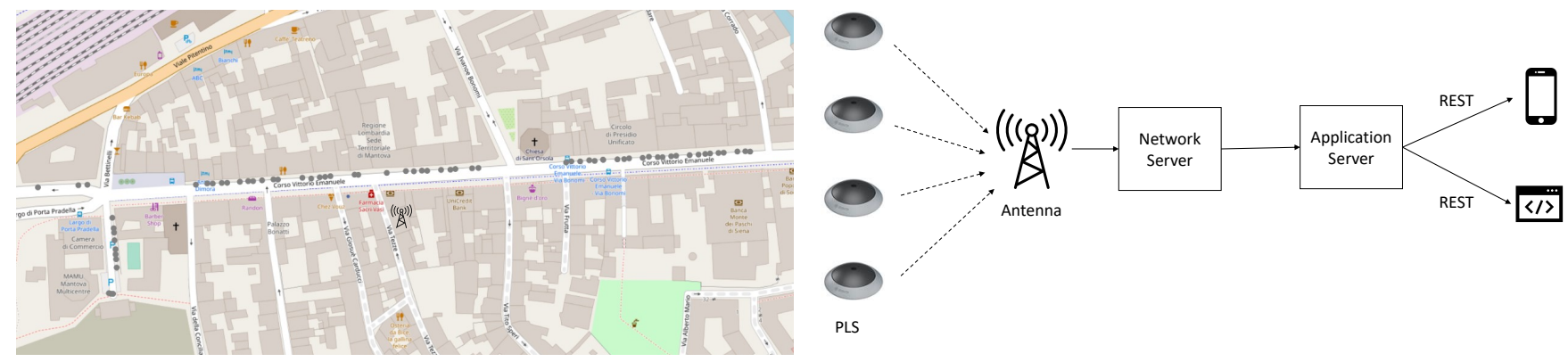

Fig. 1: Map of the deployment area in the city center of Mantova (along a straight road). 70 Parking Lot sensors have been deployed. They are represented as grey dots on the map. The LoRaWAN gate is also represented (left). The data collection infrastructure used is also represented. Sensors communicate via MQTT over LoRaWAN with the Network Server. Data are then pushed to registered clients (right).

Once received on our application server, MQTT packets are stored in a MySQL database. The key fields comprise: a timestamp, a sensor id, a sequence number for recognizing duplicate messages, an id for recognizing the type of message, and a value describing the most recent status of the sensor. MQTT packets are then used for feeding the prediction algorithms. Nevertheless, predictions are generated on-request, or fetched from a cache, whenever a REST API is queried. The API can be either used directly as a web service, or used for integrating predictions within third-party information systems.

\section{Lot Availability Prediction}

Figure 2 details the behavior of the system in time. In particular, the daily distribution of free parking spots by weekday is reported. The dark area represents the distance, expressed in free parking spots, between the first and third quartiles during the observed period. The whiskers extend to the minimum and maximum values computed without including any outliers, which are represented with single points. The chart shows a reasonably predictable behaviour in that the distance between the first and third quartile is frequently narrower than $7 \%$. The narrower the distance, the more constant the behaviour week over week.

Furthermore, Figure 2-right shows the daily trend in terms of free parking spots. The chart has been obtained by averaging, for the 10 weeks of the study, the number of free spots of a given hour and a given day. Working days and weekends are clearly differentiated. More specifically, the number of free spots increases during weekend mornings with respect to the working days, while from midday the behaviour is quite similar during the whole week. This behaviour highlights the commercial nature of the studied area.

For predicting the parking occupancy rate, two different algorithms have been studied. The first, aimed at providing us with a baseline, is based on statistics and, thus, only capable of learning the average behaviour of the system; the latter based on deep learning techniques has been developed for learning more complex behaviours. For assessing the two alternatives, the data set has been split in two sets for training and testing comprising $80 \%$ and $20 \%$ of the data respectively. More specifically, the training set contains about 28,000 messages (from 2019-12-16 to 2020-02-09) while the testing set contains about 7,000 records (from 2020-02-10 to 2020-02-23).

\section{A. Statistical Prediction}

For each day of the week and each hour of the day, the statistical model records the median of all the observed samples. Figure 3 shows how the approach works. The blue line is the median computed over the 8 weeks used as training set, while the gray and light gray areas represent a tolerable error of $\pm 3 \%$ and $\pm 4 \%$ spots respectively. The colored points (i.e., different weeks shown with different colors) represent the number of free spots actually observed in the testing set at a specific day and hour. The accuracy value is computed by dividing the number of points contained within the gray areas over the total number of points.

Given the reasonably predictable behaviour, this approach reaches $67 \%$ and $81 \%$ of accuracy when considering acceptable an error of $\pm 3 \%$ and $\pm 4 \%$ respectively. However, it is worth emphasizing how this approach is unable to correctly forecast circumstances in which the number of free spots unexpectedly moves away from the median value, as represented by the green dots in the upper right. The use of neural networks described below stems from this limitation.

\section{B. Neural Network Prediction}

The network architecture we investigated is mostly based on CNN and LSTM layers. CNN layers have been chosen for their capability of automatic feature extraction and their invariance to shifts and distortions. Due to this, local correlations can be extracted also in case of perturbations which may occur.

The chosen architecture is composed by one 1D CNN layer, one LSTM layer and three Fully Connected layers. The CNN layer is composed by 60 filters with a kernel size of 6 , the LSTM layer is composed by 1,000 cells, and the three FC layers are composed of 300, 200 and 100 neurons respectively. There is, lastly, a final FC layer composed of 8 neurons for extracting the required features. For avoiding overfitting, early stopping and dropout $=0.08$ have been applied between CNN, LSTM, and FC layers. 

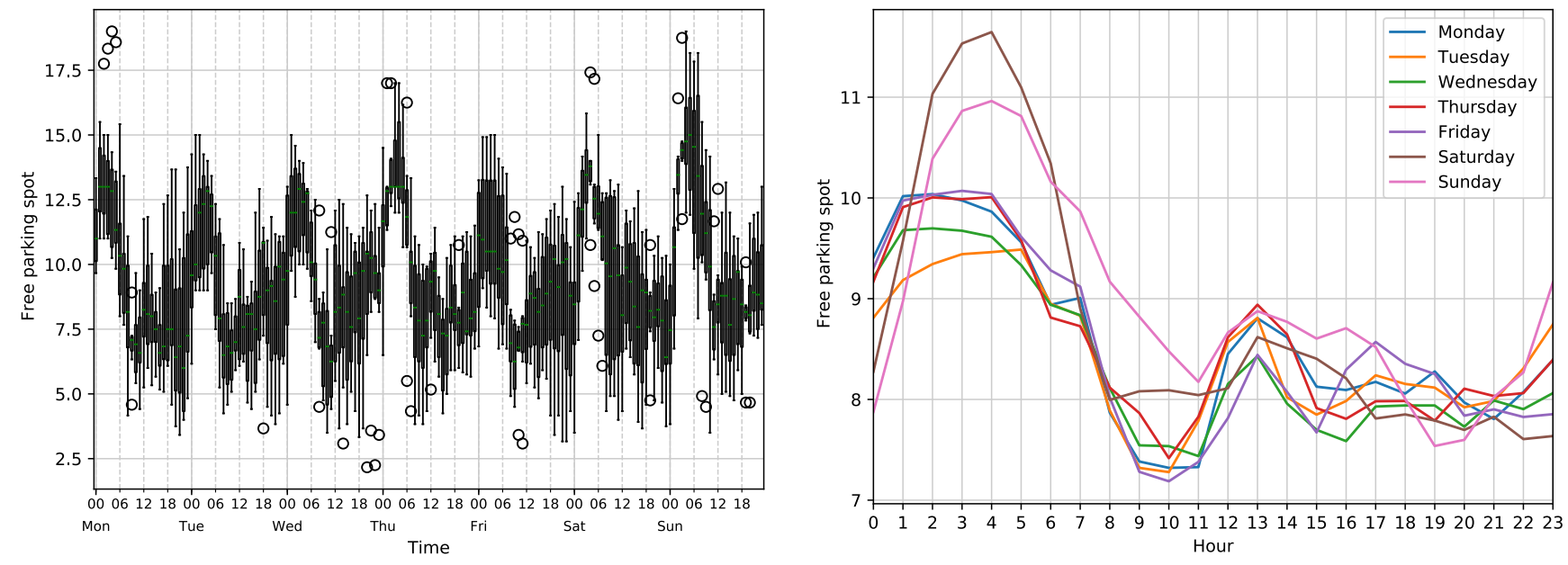

Fig. 2: Free parking spots by weekday. The boxplot shows how the data are distributed: the box indicates the data between the first and third quartiles (left). Free parking spots by hour. Each line is a day of the week, there is a different behaviour in working day and weekend day mornings (right).

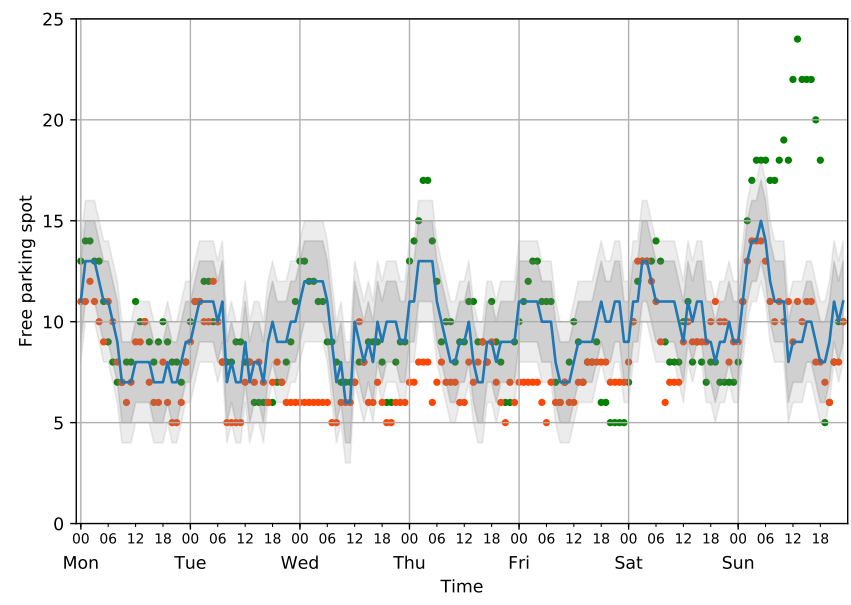

Fig. 3: Prediction based on the statistical median. The blue line represents the median number of free parking spots during the 8 weeks used as training set. The gray and light gray areas represent a band of $\pm 3 \%$ and $\pm 4 \%$ from the median, respectively. The colour of the points represents a specific week of the testing set.

Figure 4 shows the approach acting on 1 week of the testing set. The dashed lines represent the predicted values and the cyan areas represent an acceptable error of $\pm 3 \%$. The orange line represents the ground truth.

The architecture has been tested in 4 different configurations summarized in Table I: (a) both with and without the LSTM layer and $(b)$ for predicting either 1 hour ahead or 8 hours ahead. For each column of the table (representing one data set), the first value refers to the accuracy of the network itself and represents the ratio between the number of output values contained within the acceptable bounds (e.g. $\pm 3 \%$ ) and the total number of output values. The second value, instead, is calculated as the ratio between the number of measured values lying within the acceptable area and the total number of the measured values.

Experiments showed that the more distant the future to predict, the less accurate results the prediction. This is reasonable in that, as time passes after the prediction, small events integrate their effects making the final outcome more uncertain. As a general rule, shifting the horizon of predictions from 1 to 8 hours ahead implies a reduction in accuracy around $5-10 \%$. Furthermore, we observed how the introduction of the LSTM layer is beneficial. This could be explained in that future states of the system are not only tied to individual previous states but also to their history over time. Specifically, we observed that the introduction of the LSTM layer implies on average a 10-40\% improvement in prediction accuracy. More specifically, the 8 hours ahead prediction without LSTM cells (worst case) reaches $51 \%$ of accuracy with an acceptable error of $\pm 3 \%$ while the 1 hour ahead prediction with LSTM cells (best case) reaches $83 \%$. These two results become, respectively, $77 \%$ and $96 \%$ when accepting an error of $\pm 4 \%$.

\section{Birmingham Public Data Set}

For the sake of testing the generality of the architecture, we tested it on an well known public data set. We have chosen the data set Parking in Birmingham. This data set has some flaws that has been addressed using the same approach described in [13]. One specific parking area, named OthersCCCPS202, has been chosen for conducting experiments. It has a capacity of 2937 parking spots and its occupancy over time is described with 1312 records. Because of the significant difference between this parking area and the one studied in Mantova the network has been re-trained. Figure 4-right shows the predictions on 1 week of the testing set. 

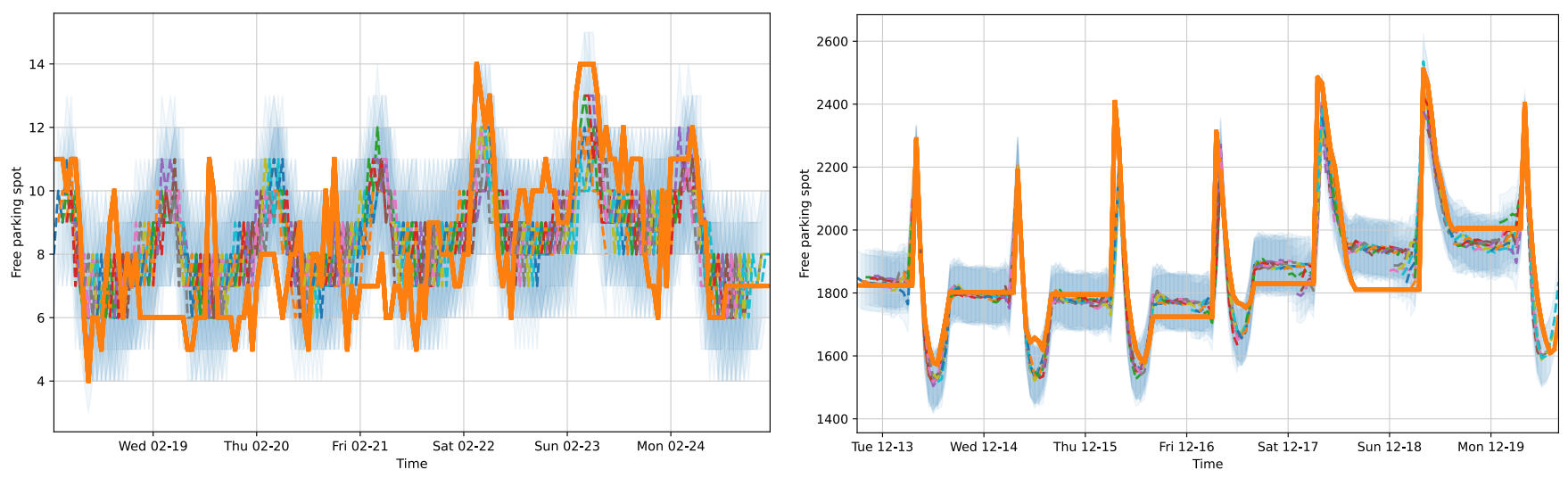

Fig. 4: 8-hours-ahead NN prediction for both the Mantova (left) and Birmingham (right) data sets. The orange lines are the real values, the dashed lines are the predicted values, the cyan areas are the errors of the prediction ( $\pm 3 \%$ spots).

TABLE I: Prediction accuracy of the studied architectures compared with the statistical prediction (baseline) on both data sets (Mantova and Birmingham).

\begin{tabular}{|l|l|l|}
\hline & Mantova & Birmingham \\
\hline MEDIAN $( \pm 3 \%)$ & $-/ 0.67$ & $-/ 0.71$ \\
\hline MEDIAN $( \pm 4 \%)$ & $-/ 0.81$ & $-/ 0.85$ \\
\hline CNN+FC $( \pm 3 \%)$ (1-hour) & $0.55 / 0.58$ & $0.73 / 0.85$ \\
\hline CNN+FC $( \pm 3 \%)$ (8-hours) & $0.55 / 0.51$ & $0.73 / 0.85$ \\
\hline CNN+LSTM+FC ( $\pm 3 \%)$ (1-hour) & $0.72 / 0.83$ & $0.86 / 0.84$ \\
\hline CNN+LSTM+FC ( $\pm 3 \%)$ (8-hours) & $0.72 / 0.76$ & $0.86 / 0.80$ \\
\hline CNN+FC $( \pm 4 \%)$ (1-hour) & $0.80 / 0.80$ & $0.72 / 0.91$ \\
\hline CNN+FC $( \pm 4 \%)$ (8-hours) & $0.80 / 0.77$ & $0.72 / 0.86$ \\
\hline CNN+LSTM+FC ( $\pm 4 \%)$ (1-hour) & $0.84 / 0.96$ & $0.81 / 0.86$ \\
\hline CNN+LSTM+FC ( $\pm 4 \%)$ (8-hours) & $0.84 / 0.93$ & $0.81 / 0.83$ \\
\hline
\end{tabular}

Experiments showed results similar to those observed in Mantova. This data set also confirms the decrease in accuracy while increasing the temporal horizon of the prediction: a difference in prediction accuracy around $5 \%$ has been observed between 1 and 8 hours ahead. Furthermore, a comparative analysis between the two data sets suggests that the relevance of the LSTM layer is not universal for this task but depends on the nature of the parking space. As an example, in this data set an improvement around 5\% has been observed when removing the LSTM. Furthermore, the network performs much more uniformly than in Mantova. It is worth noticing that the LSTM layer provides the most significant improvements in accuracy only where the $\mathrm{CNN}+\mathrm{FC}$ does not performs well. The higher the accuracy obtained with the $\mathrm{CNN}+\mathrm{FC}$ configuration, the smaller the improvement provided by LSTM layer.

As a concluding remark, our approach showed a significant generalization capability. In fact, set aside the need of retraining the network, the configuration CNN+LSTM+FC performs uniformly (about $80 \%$ accuracy) with both data sets.

\section{ForeCASting-enabled SERVICES}

The results computed by the application server are exposed via a REST API using JSON formatted responses. This API can be integrated in different systems to provide information about parking occupancy. A various number of requests can be made about past and present occupancy, median, and neural network predictions.

This API has enabled a range of novel applications and services. On the one hand, results have been integrated in parking guidance and information signs - PGI systems - (see Figure 5a). The number of available parking lots - for the parking being instrumented - does not represent the current availability, but the predicted one after the time needed to reach the parking spot (estimated on the basis of signs' location). This kind approach solves one of the main issues associated with parking guidance and information signs. Results from a recent survey [14] shows that $17 \%$ of the drivers suspected the accuracy of the information provided by such systems for the reason that the information presented by signs is not timely updated and does not reflect the situation that will be present at the time of arrival. Our approach goes exactly in the direction of addressing this concern.

On the other hand, a mobile application has been developed to show how our system can be integrated. The application allows to search for a parking calling our API for our sensored parking, while reverting to OpenStreetMaps API for normal parkings. Our application server response contains some generic information about the parking, the occupancy of the past 24 hours, the number of free spots and the predicted occupancy in the next 8 hours. In case of numbered parking lots the application can assign the specific lot where the vehicle must be parked and gives the indication to reach it. Figures $5 \mathrm{~b}$ shows a screenshot of the application. Future version of the application will try to integrate a navigation system with forecast parking lot availability. This will take into consideration the time taken to find a empty parking lot and will recommend final destinations on the basis of where parking will be available.

In addition to applications and services targeting citizens, the analysis of time-series collected by the system can enable local authorities to enact novel policies and services. Precise information about the occupancy of parking lots is an often 


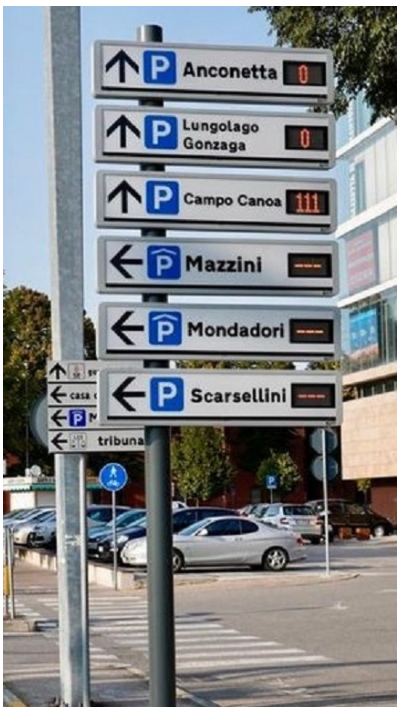

(a)

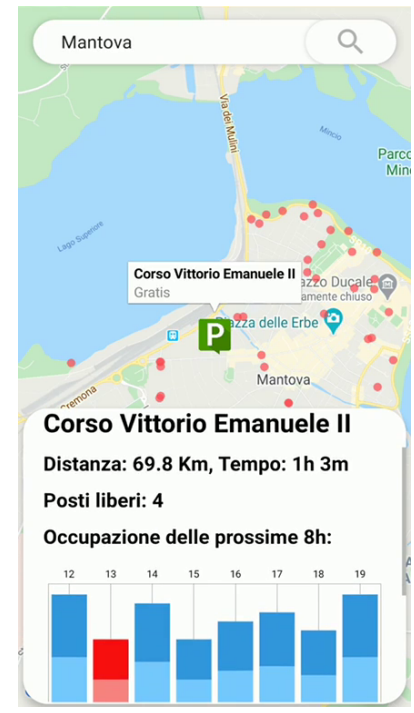

(b)
Fig. 5: Urban parking guidance and information signs integrated with the REST API $(a)$. App screen showing normal parking spaces (red points) and our smart parking space (green icon) with availability predictions below. The red prediction relates to the expected arrival time of the user (user interface in Italian) $(b)$.

lacking, but critical information to set the price of parking, or to set the price for transforming (selling) a public parking to a private one. Data already collected by our system, for training the forecasting algorithm, can naturally fulfill this information need. In addition, on the basis of such information, it could be possible to enact innovative dynamic pricing models for parking lots taking into account predicted traffic flows and parking demand. These models, other than possibly increment the profitability of parking providers, can also nudge toward traffic reduction (e.g., discouraging travel to crowded areas because parking becomes expensive), and more in general direct to more sustainable behaviors.

\section{RELATED WORK}

Parking is a large subject, as a consequence this section focuses more on the areas related to the objectives of this paper. For a more complete survey of the field, concerning all aspects from hardware sensors to IoT and cloud infrastructures, until machine learning techniques please refer to [2], [3].

Focusing on the prediction aspects, the first approaches appeared around mid 2010s, assume that cars follow a Poisson distribution when arriving at car parks and then use a Markov Chain to predict the amount of available lots [15], [16]. More recent studies on realistic car park data sets collected during the Smart Santander Initiative showed that the Weibull distribution is more accurate for describing parking periods of different parking areas [10]. In [17], a smart parking system for shopping malls capable of predictive features has been introduced in Abu Dhabi. In [12], the authors have developed a self-regressive model space-time vector that can be used to predict the evolution of the availability of parking for onstreet and off-street parking at the driver's expected arrival time. The project takes into account temporal correlations in parking availability as well as spatial correlations. It is used to recommend the parking location having the highest probability. They used real-time parking data from the San Francisco area to evaluate the results and verify the model. Data collected in San Francisco have been also used for comparing spatio-temporal clustering strategies [18] aiming at reducing the storage requirements of prediction systems while keeping similar accuracy levels. In [11], regressions, neural networks, and support vector machines have been compared for predicting car park occupancy rates, while Rajabioun et. al. [12] applied a multi-variate regressive model for predicting temporal and spatial correlations of parking lots availability.

Deep neural networks have been initially applied for predicting car park availability by applying feed-forward networks and, more recently, showed a sensible improvement in accuracy when coupled with IoT systems continuously providing new data and back-propagation [10]. Xiang et. al. [19] proposed an approach for detecting real-time parking occupancy at gas stations using Haar-AdaBoosting and CNNs. Stolfi et. al [13], proposed a study of parking occupancy data in order to test several forecasting strategies such as polynomial adjustment, fourier series, $\mathrm{k}$-means grouping and time series. All the alternatives showed similar forecasting capabilities. Camero et. al. [9], recently presented a technique based on deep learning with recurrent neural networks to forecast the parking occupancy rate. The paper also proposes to use the recently introduced concept of NeuroEvolution for optimizing the neural network used for processing parking data. The models built in these works however focus mainly on prediction neglecting emerging technologies such as IoT that can be used for further improvements; which led to rest of this work. Overall, in both data sets, the proposed CNN+LSTM+FC network achieves state of the art results improving over the majority of the works in the literature.

From a more general perspective, early works, such as [20], started with the evaluation of different types of sensors (acoustics, light, magnetic) for the sake of parking management. Data collected from sensors were sent to a single server and centrally processed. In [21], a comprehensive analysis of the most relevant aspects of the design of a smart parking system has been presented. The article focuses mainly on the selection of adequate sensors and how to deploy them for accurately detecting state changes in the parking lot. Other authors such as [22] developed prototypes using RFID and IoT to detect the car details and then used IR sensor to find the presence of the car so that all details are accessed remotely through the cloud. The same RFID-based technique has also been tested by Pawowicz et al. [23] to improve traffic management in a smart city environment. However, the prediction aspects has not been addressed with this technology. Another case study [24] introduced an automated valet for parking based on hybrid robotic arms. The robotic helps to optimize the usage of 
parking space and make use of Deep Q-Learning as a learning method. Amato et. al. [6] proposed an intelligent parking system based on artificial vision. The system uses a camera and deep learning algorithms to determine the availability of spaces in a parking lot. The system is then compared with two prior methods existing in literature such as PKLot [25] and CNRPark-EXT [5]. Mago et. al. [26] proposed another model for designing a parking management system based on video processing techniques. The proposed method detects available parking lots in real-time using cameras and assign vehicles entering the area to specific places. Both these proposals deal with real-time, vision-based detection but still neglect predictive aspects.

\section{CONCLUSION}

In this paper we have presented an architecture for the smart parking domain in which we integrate a new method for predicting available parking spaces with a novel combination of smart city technologies, IoT and machine learning still overlooked in literature. More specifically, 70 Parking Lot sensors have been deployed in the city center of a mid-sized Italian city. The data collection architecture has been built around a LoraWAN-compliant network server acquiring data via a single physical gate over a radius of about $5 \mathrm{~km}$. This experience showed how the LoRaWAN technology is well suited for IoT applications requiring long-term running times without recharging and low bandwidth. It also showed the relevance of corporate operations that need to take into account peculiar weather conditions and unusual events to guarantee long lasting large-scale deployments. Finally, experiments showed that deep learning techniques can be successfully used for predicting the availability of parking spaces within a 8 hours time horizon.

\section{ACKNOWLEDGMENT}

This work has been supported by Robert Bosch GmbH. The authors would like to thank Dr. Giulio Lancellotti and Dr. Piepaolo Palazzoli for their vision and guidance in the industrial portion of the field and Denise Landini and Alessandro Lambertini for contributing to the development of a supporting mobile app. Special thanks also to the A2A Group, CellNext, Aster and Comune di Mantova for their support in the deployment and operation of the system.

\section{REFERENCES}

[1] Y. Zhang and A. Haghani, "A gradient boosting method to improve travel time prediction," Transportation Research Part C: Emerging Technologies, vol. 58, pp. 308-324, 2015.

[2] F. Al-Turjman and A. Malekloo, "Smart parking in iot-enabled cities: A survey," Sustainable Cities and Society, vol. 49, p. 101608, 2019.

[3] T. Lin, H. Rivano, and F. L. Mouel, "A survey of smart parking solutions," IEEE Transactions on Intelligent Transportation Systems, vol. 18, 2017.

[4] F. Zantalis, G. Koulouras, S. Karabetsos, and D. Kandris, "A review of machine learning and iot in smart transportation," Future Internet, vol. 11, no. 4, p. 94, 2019.

[5] G. Amato, F. Carrara, F. Falchi, C. Gennaro, and C. Vairo, "Car parking occupancy detection using smart camera networks and deep learning," in 2016 IEEE Symposium on Computers and Communication (ISCC). IEEE, 2016, pp. 1212-1217.
[6] G. Amato, F. Carrara, F. Falchi, C. Gennaro, C. Meghini, and C. Vairo, "Deep learning for decentralized parking lot occupancy detection," Expert Systems with Applications, vol. 72, pp. 327-334, 2017.

[7] R. Ke, Y. Zhuang, Z. Pu, and Y. Wang, "A smart, efficient, and reliable parking surveillance system with edge artificial intelligence on iot devices," IEEE Transactions on Intelligent Transportation Systems, pp. 1-13, 2020.

[8] C. Zhu, A. Mehrabi, Y. Xiao, and Y. Wen, "Crowdparking: Crowdsourcing based parking navigation in autonomous driving era," in 2019 International Conference on Electromagnetics in Advanced Applications (ICEAA), 2019, pp. 1401-1405.

[9] A. Camero, J. Toutouh, D. H. Stolfi, and E. Alba, "Evolutionary deep learning for car park occupancy prediction in smart cities," in International Conference on Learning and Intelligent Optimization. Springer, 2018, pp. 386-401.

[10] E. I. Vlahogianni, K. Kepaptsoglou, V. Tsetsos, and M. G. Karlaftis, "A real-time parking prediction system for smart cities," Journal of Intelligent Transportation Systems, vol. 20, no. 2, pp. 192-204, 2016.

[11] Y. Zheng, S. Rajasegarar, and C. Leckie, "Parking availability prediction for sensor-enabled car parks in smart cities," in 2015 IEEE Tenth International Conference on Intelligent Sensors, Sensor Networks and Information Processing (ISSNIP). IEEE, 2015, pp. 1-6.

[12] T. Rajabioun and P. A. Ioannou, "On-street and off-street parking availability prediction using multivariate spatiotemporal models," IEEE Transactions on Intelligent Transportation Systems, vol. 16, no. 5, pp. 2913-2924, 2015.

[13] D. H. Stolfi, E. Alba, and X. Yao, "Predicting car park occupancy rates in smart cities," in International Conference on Smart Cities. Springer, 2017, pp. 107-117.

[14] Z. Liu, W. Deng, and D. Pan, "Driver response to parking guidance and information systems in nanjing," in International Conference of Chinese Transportation Professionals (ICCTP), Nanjing, China, 2011.

[15] A. Klappenecker, H. Lee, and J. L. Welch, "Finding available parking spaces made easy," Ad Hoc Networks, vol. 12, pp. 243-249, 2014.

[16] S. Pullola, P. K. Atrey, and A. El Saddik, "Towards an intelligent gps-based vehicle navigation system for finding street parking lots," in 2007 IEEE International Conference on Signal Processing and Communications. IEEE, 2007, pp. 1251-1254.

[17] S. A. Alkheder, M. M. Al Rajab, and K. Alzoubi, "Parking problems in abu dhabi, uae toward an intelligent parking management system "adip: Abu dhabi intelligent parking"," Alexandria Engineering Journal, vol. 55, no. 3, pp. 2679-2687, 2016.

[18] F. Richter, S. Di Martino, and D. C. Mattfeld, "Temporal and spatial clustering for a parking prediction service," in 2014 IEEE 26th International Conference on Tools with Artificial Intelligence. IEEE, 2014, pp. 278-282.

[19] X. Xiang, N. Lv, M. Zhai, and A. El Saddik, "Real-time parking occupancy detection for gas stations based on haar-adaboosting and cnn," IEEE Sensors Journal, vol. 17, no. 19, pp. 6360-6367, 2017.

[20] R. Kumar, N. K. Chilamkurti, and B. Soh, "A comparative study of different sensors for smart car park management," in the 2007 International Conference on Intelligent Pervasive Computing (IPC 2007). IEEE, 2007, pp. 499-502.

[21] M. Bachani, U. M. Qureshi, and F. K. Shaikh, "Performance analysis of proximity and light sensors for smart parking," Procedia Computer Science, vol. 83, pp. 385-392, 2016.

[22] B. K. Gandhi and M. K. Rao, "A prototype for iot based car parking management system for smart cities," Indian Journal of Science and Technology, vol. 9, no. 17, pp. 1-6, 2016.

[23] B. Pawłowicz, M. Salach, and B. Trybus, "Infrastructure of rfidbased smart city traffic control system," in Conference on Automation. Springer, 2019, pp. 186-198.

[24] N. Shoeibi and N. Shoeibi, "Future of smart parking: Automated valet parking using deep q-learning," in International Symposium on Distributed Computing and Artificial Intelligence. Springer, 2019, pp. 177-182.

[25] P. R. De Almeida, L. S. Oliveira, A. S. Britto Jr, E. J. Silva Jr, and A. L. Koerich, "Pklot-a robust dataset for parking lot classification," Expert Systems with Applications, vol. 42, no. 11, pp. 4937-4949, 2015.

[26] N. Mago and S. Kumar, "A machine learning technique for detecting outdoor parking," International Journal of Engineering \& Technology, vol. 7, no. 2.30 , pp. 39-43, 2018. 